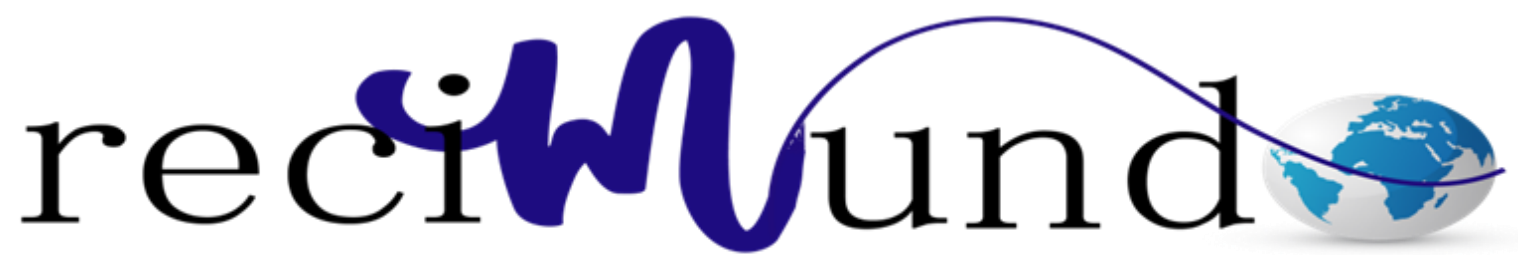

Revista Científica Mundo de la Investigación y el Conocimiento

\author{
Víctor Hugo Verdezoto Vargas a; Gonzalo Isaías Almagro Mayorga b; Iván \\ Edmundo Ramos Mosquera ${ }^{c}$; Ángel Polivio Jines Carrasco ${ }^{\text {d; }}$ Diego Rivera ${ }^{\mathrm{e}}$
}

Las sustancias húmicas como sustituto de la materia orgánica en cultivo de rosas

(Rosa spp.)

Revista Científica Mundo de la Investigación y el Conocimiento. Vol. 1 núm., 5, diciembre, ISSN: 2588-073X, 2017, pp. 995-1010

DOI: 10.26820/recimundo/1.5.2017.995-1010

Editorial Saberes del Conocimiento

Recibido: 05/08/2017

Aceptado: 10/40/2017

a. Docentes de la Facultad de Ciencias Agrarias de la Universidad de Guayaquil; victor.verdezotov@ug.edu.ec

b. Docentes de la Facultad de Ciencias Agrarias de la Universidad de Guayaquil; gonzalo.almagrom@ug.edu.ec

c. Docentes de la Facultad de Ciencias Agrarias de la Universidad de Guayaquil; ivan.ramosm@ug.edu.ec

d. Docentes de la Facultad de Ciencias Agrarias de la Universidad de Guayaquil; angel.jinesc@ug.edu.ec

e. Ingeniero Agrónomo Zamorano. Técnico Plantación EXFLODEC. 


\section{Las sustancias húmicas como sustituto de la materia orgánica en cultivo de rosas (Rosa spp.)}

Vol. 1, núm. 5., (2017)

Víctor Hugo Verdezoto Vargas; Gonzalo Isaías Almagro Mayorga; Iván Edmundo Ramos Mosquera; Ángel Polivio Jines Carrasco; Diego Rivera

\section{RESUMEN}

La Leonardita es una sustancia vegetal humificada, muy rica en materia orgánica, está constituida principalmente de carbono (55\% en peso), y materiales húmicos (moléculas orgánicas complejas como Carboxilos, Hidroxilos y Carbonilos). Este tipo de materiales se utilizan para la extracción de ácidos húmicos y fúlvicos, componentes principales de las sustancias húmicas. El objetivo del presente estudio fue determinar el efecto de las sustancias húmicas (Humiplex Plus) sobre el cultivo de rosas. El experimento se desarrolló en una finca perteneciente a la parroquia Otón del cantón Cayambe ubicada en la provincia de Pichincha; el cual tuvo lugar bajo invernadero en camas de 30 metros de largo por un metro de ancho a una altura de 10 a $15 \mathrm{~cm}$. sobre el nivel del suelo; en el diseño estadístico se aplicaron seis tratamientos con cuatro repeticiones. De los resultados obtenidos, se concluye que con la aplicación de 160 gramos (T5) de sustancias húmicas (Humiplex Plus)/m2 en camas de cultivo de rosas, se obtuvo un número de basales superior en 1.63, un número de medias piernas superior en 1.08 y una altura de plantas superior en $9.9 \mathrm{~cm}$, frente al testigo, cifras que indican que a medida que se incrementa la dosis de sustancias húmicas (Humiplex Plus/m2 de suelo) enriquecida con micro elementos (Fe, $\mathrm{Zn}, \mathrm{Mg}, \mathrm{Mn}, \mathrm{Cu}$, Bo y S), se estimulan el crecimiento y desarrollo de varios órganos de la planta tratada.

Palabras Clave: Ácidos húmicos, basales, invernadero, leonardita y rosas. 


\title{
Las sustancias húmicas como sustituto de la materia orgánica en cultivo de rosas (Rosa spp.)
}

Vol. 1, núm. 5., (2017)

Víctor Hugo Verdezoto Vargas; Gonzalo Isaías Almagro Mayorga; Iván Edmundo Ramos

Mosquera; Ángel Polivio Jines Carrasco; Diego Rivera

\begin{abstract}
Leonardite is a humified plant substance, very rich in organic matter, mainly composed of carbon (55\% by weight), and humic materials (complex organic molecules such as Carboxyls, Hydroxyls and Carbonyl). This type of material is used for the extraction of humic and fulvic acids, the main components of humic substances. The objective of the present study was to determine the effect of humic substances (Humiplex Plus) on the cultivation of roses. The experiment was carried out on a farm belonging to the Otón parish of the Cayambe canton located in the province of Pichincha; which took place under greenhouse in beds 30 meters long by one-meter-wide at a height of 10 to $15 \mathrm{~cm}$. above ground level; in the statistical design six treatments with four repetitions were applied. From the results obtained, it is concluded that with the application of 160 grams (T5) of humic substances (Humiplex Plus) / $\mathrm{m} 2$ in beds of cultivation of roses, a higher number of basal was obtained in 1.63, a number of upper legs in 1.08 and a plant height higher by $9.9 \mathrm{~cm}$, compared to the control, figures that indicate that as the dose of humic substances increases (Humiplex Plus / $\mathrm{m} 2$ of soil) enriched with micro elements ( $\mathrm{Fe}, \mathrm{Zn}, \mathrm{Mg}, \mathrm{Mn}, \mathrm{Cu}, \mathrm{Bo}$ and $\mathrm{S}$ ), the growth and development of various organs of the treated plant are stimulated.
\end{abstract}

Key words: humic acids, basal, greenhouse, leonardite and roses. 


\section{Las sustancias húmicas como sustituto de la materia orgánica en cultivo de rosas (Rosa spp.)}

Vol. 1, núm. 5., (2017)

Víctor Hugo Verdezoto Vargas; Gonzalo Isaías Almagro Mayorga; Iván Edmundo Ramos Mosquera; Ángel Polivio Jines Carrasco; Diego Rivera

\section{Introducción.}

Actualmente la tendencia a utilizar productos orgánicos, permiten a los agricultores obtener mayores rendimientos sin alterar el ambiente y con una notable disminución de uso de fertilizantes químicos (Potisek et al., 2013).

La materia orgánica del suelo, es un conjunto de residuos orgánicos de origen animal y/o vegetal, que están en diferentes grados de descomposición, y que se acumulan tanto en la superficie como dentro del perfil del suelo. El carbono orgánico del suelo, es el principal elemento que forma parte de la materia orgánica (Janampa et al., 2014). Aporta al suelo fertilidad, así como mejora las condiciones físicas del mismo y por ende la vida microbiana, favoreciendo el desarrollo de la planta. Es fuente principal de nitrógeno, fósforo, azufre y algunos elementos menores. También aumenta la capacidad amortiguadora, y tiene gran influencia en la capacidad de intercambio catiónico del suelo (FAO, 2013). Está presente en suelos, sedimentos y puede sufrir un proceso de descomposición o estar en proceso de humificación (Pablo et al., 2015).

Las sustancias húmicas son macromoléculas complejas donde se encuentran diferentes grupos funcionales que le permiten actuar como polielectrolitos de ácidos débiles y ser sitios de reacción con diferentes agentes químicos (Huelva et al., 2013); son el producto de la actividad de los distintos grupos de organismos que incluye procesos de polimerización complejos que hacen que el producto final no tenga ninguna similitud con los residuos frescos pero que presenta atributos que confieren al suelo las propiedades que lo habilitan para albergar la vida: estructura, aireación, capacidad de retención de humedad, capacidad de intercambio de iones, resistencia a las condiciones ambientales (Álvaro, 2008). Las sustancias húmicas favorecen el desarrollo 


\section{Las sustancias húmicas como sustituto de la materia orgánica en cultivo de rosas (Rosa spp.)}

Vol. 1, núm. 5., (2017)

Víctor Hugo Verdezoto Vargas; Gonzalo Isaías Almagro Mayorga; Iván Edmundo Ramos

Mosquera; Ángel Polivio Jines Carrasco; Diego Rivera

vegetativo, promoviendo el crecimiento de la parte aérea y raíces (Bettoni et al., 2016), ya que tienen profundos efectos físicos, químicos y biológicos sobre el suelo, con un efecto estimulante para el crecimiento de las plantas; influyen en la movilidad de compuestos orgánicos no iónicos como pesticidas y contaminantes, removiéndolos de las soluciones acuosas (Reyes et al., 2017) y son aplicados al suelo para desbloquear los elementos nutritivos y ponerlos a disposición de las raíces de las plantas (Túqueres, 2013).

Las sustancias húmicas han sido ampliamente reconocidas como un promotor de crecimiento de plantas principalmente por cambios en la arquitectura de la raíz y su dinámica de crecimiento, dando como resultado un mayor tamaño de raíz, ramificación y / o una mayor densidad del vello radicular con mayor área de superficie (Canellas y Olivares, 2014); son de gran importancia por el efecto que ejercen sobre la actividad enzimática en las plantas, que les posibilita mitigar el estrés oxidativo que se genera por el déficit de agua (Hernández et al., 2012); y, juegan un papel importante en las interacciones bióticas-abióticas de la raíz y suelo que contribuye a la adaptación de la planta a ambientes externos (Kulikova et al., 2016).

Los ácidos húmicos, son sustancias coloidales con un alto grado de humificación y estructura compleja, que contienen alrededor de 50 a $62 \%$ de carbono. Estos ácidos son producto de la humificación del material orgánico fosilizado, como turbas y lignitos que provienen de minas de carbón (Gutiérrez et al., 2015); mantienen la estabilidad de la reacción del suelo, la adsorción / fijación / quelato de cationes, lo que aumenta la disponibilidad de agua y nutrientes para las plantas (Mindari et al., 2014); se obtienen de diferentes fuentes, como suelo, humus, turba, lignito oxidado y el carbón; puede presentar directamente efectos positivos sobre el crecimiento de la planta 


\section{Las sustancias húmicas como sustituto de la materia orgánica en cultivo de rosas (Rosa spp.)}

Vol. 1, núm. 5., (2017)

Víctor Hugo Verdezoto Vargas; Gonzalo Isaías Almagro Mayorga; Iván Edmundo Ramos Mosquera; Ángel Polivio Jines Carrasco; Diego Rivera

incrementando su desarrollo, en los brotes, raíces, permitiendo mejor absorción de nitrógeno, potasio, calcio, magnesio y fósforo (Ayón et al., 2017); facilitan el crecimiento de las plantas al mejorar la absorción de nutrientes por sus efectos hormonales (Chang et al., 2012); se encuentran disponibles en forma líquida, polvo o gránulos, se utilizan en cultivos a campo abierto y en las áreas protegidas y como bioestimulantes de plantas y mejoradores del suelo (Manda et al., 2014).

Los ácidos fúlvicos son fracciones húmicas que permanecen en la solución acuosa acidificada, soluble en ácidos y bases. Es pardo-amarillento, de menor peso molecular (900-5000 D) y posee cerca de $43-52 \%$ de carbono (Velasco et al., 2016).

Por la importancia que representa las sustancias húmicas en el acondicionamiento de la calidad de suelo para mejorar la nutrición vegetal y producción agrícola; esta investigación tuvo como propósito determinar su efecto (Humiplex Plus) en el incremento del número de brotes basales, medias piernas y altura de plantines de rosas.

\section{Materiales y Métodos.}

La Plantación EXFLODEC del grupo FLORINSA, se encuentra ubicada en la Parroquia Otón, al sur del cantón Cayambe en la provincia de Pichincha, cuyas coordenadas geográficas son de $0^{\circ} 3^{\prime} 14^{\prime \prime}$ de latitud sur y $78^{\circ} 8^{\prime} 22^{\prime \prime}$ de longitud oeste. Cuenta con una superficie aproximada de $24.45 \mathrm{~km} 2$, ocupando el $94 \%$ de su territorio. Su temperatura media anual de $15.1^{\circ} \mathrm{C}$, cuyos valores medios de precipitación multianual es de 838.4 mm y la evapotranspiración anual de 706 mm; se encuentra a una altitud entre los 2400 y $3200 \mathrm{msnm}$. El $2.48 \%$ de territorio está constituido por 


\section{Las sustancias húmicas como sustituto de la materia orgánica en cultivo de rosas (Rosa spp.)}

Vol. 1, núm. 5., (2017)

Víctor Hugo Verdezoto Vargas; Gonzalo Isaías Almagro Mayorga; Iván Edmundo Ramos Mosquera; Ángel Polivio Jines Carrasco; Diego Rivera

suelo areno-franco, el $43.83 \%$ suelo franco, el $6.53 \%$ suelo franco-arcillo-arenoso, el $46.52 \%$ suelo franco-arenoso; el 0.63\% no aplica (GAD Otón, 2014).

El cultivo de rosas se maneja bajo invernadero, en camas mediante un sistema de fertirrigación, que permite mantener una temperatura estable durante todo el año. Las sustancias húmicas, se aplicaron por una sola vez al momento de la preparación de las camas de cultivo, mezclando el sustrato (suelo) con las sustancias húmicas; a continuación, se trasplantaron las plantas de rosas de la variedad Cadillac, en estado de trasplante. Las camas para el cultivo de rosas fueron de 30 metros de largo por un metro de ancho y a una altura sobre el nivel del suelo de $10 \mathrm{a}$ $15 \mathrm{~cm}$.

En este experimento, se investigaron los efectos de los ácidos húmicos como estimulantes de la emisión de brotes basales, medias piernas y altura de plantas en conjunción con una marcada proliferación de sitios de emergencia de las raíces laterales. Las dosis de sustancias húmicas aplicadas a las camas de cultivo de rosas en estado de plantines, fueron las siguientes:

Tabla 1. Número de tratamientos

\begin{tabular}{llc}
\hline $\mathrm{N}^{\circ}$ & Tratamiento & $\begin{array}{c}\text { Dosis } \\
\left(\mathrm{gr} / \mathrm{m}^{2}\right)\end{array}$ \\
\hline 1 & T1 $=$ Testigo & 0 \\
2 & T2 $=$ Humiplex Plus & 40 \\
3 & T3 = Humiplex Plus & 80 \\
4 & T4 = Humiplex Plus & 120 \\
5 & T5 $=$ Humiplex Plus & 160 \\
6 & T6 $=$ Humiplex Plus & 200 \\
\hline
\end{tabular}




\section{Las sustancias húmicas como sustituto de la materia orgánica en cultivo de rosas (Rosa spp.)}

Vol. 1, núm. 5., (2017)

Víctor Hugo Verdezoto Vargas; Gonzalo Isaías Almagro Mayorga; Iván Edmundo Ramos Mosquera; Ángel Polivio Jines Carrasco; Diego Rivera

En el diseño estadístico, se aplicaron seis tratamientos con cuatro repeticiones, de cada tratamiento fueron tomadas 10 plantas al azar para la obtención de las variables como número de basales, número de medias piernas y altura de plantas en centímetros, variables que fueron evaluadas a los dos meses de edad de plantación.

Para la obtención de resultados de las variables evaluadas, se utilizó el paquete estadístico "Infostat", en el cual se ingresaron los datos de cada tratamiento y repetición para obtener los resultados estadísticos e interpretación de los mismos. Para el análisis funcional, se aplicó la prueba de medias mediante Duncan al 5\% de probabilidad.

\section{Resultados}

Los tratamientos T5 y T6 (160 y 200 gramos/m2 de suelo) alcanzaron los valores más altos con 2.25 y 2.03 brotes denominados "basales" respectivamente y difiere estadísticamente con el testigo (T1) sin aplicación que tuvo un valor mínimo de 0.62 brotes (Tabla 2), con un coeficiente de variación para esta variable de $4.97 \%$.

Tabla 2. Número de brotes basales / planta

\begin{tabular}{lccc}
\hline $\mathrm{N}^{\circ}$ & $\begin{array}{c}\text { Tratamiento } \\
\left(\mathrm{gr} / \mathrm{m}^{2}\right)\end{array}$ & $\begin{array}{c}\text { Número de brotes } \\
\text { basales / planta }\end{array}$ & $\begin{array}{c}\text { Significancia } \\
(*)\end{array}$ \\
\hline $\mathrm{T} 1$ & 0 & 0.62 & $\mathrm{f}$ \\
$\mathrm{T} 2$ & $40 \mathrm{~g}$ & 0.88 & $\mathrm{e}$ \\
$\mathrm{T} 3$ & $80 \mathrm{~g}$ & 1.29 & $\mathrm{~d}$ \\
$\mathrm{~T} 4$ & $120 \mathrm{~g}$ & 1.79 & $\mathrm{c}$ \\
$\mathrm{T} 5$ & $160 \mathrm{~g}$ & 2.25 & $\mathrm{a}$ \\
$\mathrm{T} 6$ & $200 \mathrm{~g}$ & 2.03 & $\mathrm{~b}$ \\
\hline
\end{tabular}




\section{Las sustancias húmicas como sustituto de la materia orgánica en cultivo de rosas (Rosa spp.)}

Vol. 1, núm. 5., (2017)

Víctor Hugo Verdezoto Vargas; Gonzalo Isaías Almagro Mayorga; Iván Edmundo Ramos

Mosquera; Ángel Polivio Jines Carrasco; Diego Rivera

Los tratamientos T5 y T6 (160 y 200 gramos/m2 de suelo) alcanzaron los valores más altos con 1.92 y 1.79 brotes denominados "medias piernas" y difieren estadísticamente con el testigo (T1) sin aplicación que tuvo un valor mínimo de 0.84 brotes (Tabla 3). Las cifras indican que a medida que se incrementa la dosis de sustancias húmicas (Humiplex Plus) enriquecida con micro elementos (Fe, $\mathrm{Zn}, \mathrm{Mg}, \mathrm{Mn}, \mathrm{Cu}, \mathrm{Bo}$ y S), estimulan el crecimiento y desarrollo de varios órganos de la planta tratada, con un coeficiente de variación para esta variable de $8.28 \%$.

Tabla 3. Número de brotes denominados "medias piernas" / planta

\begin{tabular}{lccc}
\hline $\mathrm{N}^{\circ}$ & $\begin{array}{c}\text { Tratamiento } \\
\left(\mathrm{gr} / \mathrm{m}^{2}\right)\end{array}$ & $\begin{array}{c}\text { Número de medias } \\
\text { piernas } / \text { planta }\end{array}$ & $\begin{array}{c}\text { Significancia } \\
\left({ }^{*}\right)\end{array}$ \\
\hline $\mathrm{T} 1$ & 0 & 0.84 & $\mathrm{e}$ \\
$\mathrm{T} 2$ & $40 \mathrm{~g}$ & 0.60 & $\mathrm{~d}$ \\
$\mathrm{~T} 3$ & $80 \mathrm{~g}$ & 1.02 & $\mathrm{c}$ \\
$\mathrm{T} 4$ & $120 \mathrm{~g}$ & 1.40 & $\mathrm{~b}$ \\
$\mathrm{~T} 5$ & $160 \mathrm{~g}$ & 1.92 & $\mathrm{a}$ \\
$\mathrm{T} 6$ & $200 \mathrm{~g}$ & 1.79 & $\mathrm{a}$ \\
\hline \multicolumn{2}{l}{ Coeficiente de variación } \\
\hline
\end{tabular}

Los tratamientos T5 y T6 (160 y 200 gramos/m2 de suelo) alcanzaron los valores más altos con $36.30 \mathrm{~cm}$ y $35.60 \mathrm{~cm}$ de altura de plantas y difiere estadísticamente con el testigo (T1) sin aplicación que tuvo un valor mínimo de $26.40 \mathrm{~cm}$ (Tabla 4); estas cifras indican que a medida que se incrementa la dosis de sustancias húmicas/m2 de suelo, también se incrementa la altura de plantas en cultivo de rosas, cuyo coeficiente de variación para esta variable es de 9.83\%. 


\section{Las sustancias húmicas como sustituto de la materia orgánica en cultivo de rosas (Rosa spp.)}

Vol. 1, núm. 5., (2017)

Víctor Hugo Verdezoto Vargas; Gonzalo Isaías Almagro Mayorga; Iván Edmundo Ramos Mosquera; Ángel Polivio Jines Carrasco; Diego Rivera

Tabla 4. Altura de plantas en $\mathrm{cm}$

\begin{tabular}{lccc}
\hline $\mathrm{N}^{\circ}$ & $\begin{array}{c}\text { Tratamiento } \\
\left(\mathrm{gr} / \mathrm{m}^{2}\right)\end{array}$ & $\begin{array}{c}\text { Altura de plantas } \\
\text { en } \mathrm{cm}\end{array}$ & $\begin{array}{c}\text { Significancia } \\
\left({ }^{*}\right)\end{array}$ \\
\hline $\mathrm{T} 1$ & 0 & 26.40 & $\mathrm{c}$ \\
$\mathrm{T} 2$ & $40 \mathrm{~g}$ & 29.50 & $\mathrm{bc}$ \\
$\mathrm{T} 3$ & $80 \mathrm{~g}$ & 33.30 & $\mathrm{ab}$ \\
$\mathrm{T} 4$ & $120 \mathrm{~g}$ & 33.90 & $\mathrm{ab}$ \\
$\mathrm{T} 5$ & $160 \mathrm{~g}$ & 36.30 & $\mathrm{a}$ \\
$\mathrm{T} 6$ & $200 \mathrm{~g}$ & 35.60 & $\mathrm{a}$ \\
\hline
\end{tabular}

\section{Discusión}

González et al. (2018), aplicaron ácidos orgánicos al suelo, obteniendo mayor productividad en rosa de corte como consecuencia del incremento en la longitud de brotes y número de brotes basales, lo cual fue más evidente en la dosis de $15 \mathrm{~g}$. cama-1 del producto, que en comparación con la aplicación de Humiplex Plus en dosis de 160 g. cama-1 de 30 m2 de suelo se obtuvo 2.25 brotes basales/planta de rosa, frente al testigo que fue de 0.62 basales por planta; situación similar obtuvieron los investigadores Noriega et al. (2015), que encontraron respuestas significativas en el número de botones y flores comerciales en rosas, donde el mayor promedio 34.75 botones y 18.33 flores comerciales se obtuvo en el $\mathrm{T} 1(\mathrm{P}<0.5)$ donde se aplicó el fertilizante comercial Humifer, seguido por el T5 con lombricomposta con un promedio de 33.80 botones y 17.87 flores comerciales, lo que demuestra que hay diferencia estadística entre los ácidos húmicos comerciales y la lombricomposta que puede competir con estos. 


\section{Las sustancias húmicas como sustituto de la materia orgánica en cultivo de rosas (Rosa spp.)}

Vol. 1, núm. 5., (2017)

Víctor Hugo Verdezoto Vargas; Gonzalo Isaías Almagro Mayorga; Iván Edmundo Ramos

Mosquera; Ángel Polivio Jines Carrasco; Diego Rivera

Ramírez et al. (2011), obtuvieron una mejora en la productividad de rosas al adicionar humus de lombriz líquido a razón de $30 \mathrm{ml}$ a la fórmula de fertilización convencional empleada, lo cual aumenta la productividad por planta en el segundo ciclo del cultivo de rosa cv. Classy.

Ahmad et al. (2013), experimentaron tres aplicaciones de ácidos húmicos junto con NPK, superando todos los demás tratamientos para la mayoría de crecimiento e índices fisiológicos del gladiolo en producción y demostraron ser eficaz para mejorar el rendimiento y calidad de tallos de gladiolos cortados. Con la combinación de ácidos húmicos a partir de leonardita con la fertilización NPK, obtuvieron 20.6 cormos, 12.4 mm de diámetro y 6.3 g de peso, en relación al testigo; investigación que coincide con la publicación de Ali et al. (2014), que señalan que la adición de ácidos húmicos junto con mezclas de macronutrientes (NPK) tuvieron un efecto positivo en el crecimiento de Tulipa gesneriana L.; lo cual corrobora que los ácidos húmicos constituyen el mejor tónico de mejora del crecimiento especialmente para la industria de flores cortadas.

Baldotto y Baldotto (2015), investigaron que el tratamiento de semillas ornamentales de girasol con ácidos húmicos en dosis de 15-20 mmol0 1-1 C, aumentaron el número y calidad de tallos y flores; mientras que, Ibrahim et al. (2016), concluyen que, aplicando vía foliar ácidos húmicos, lograron aumentar la disponibilidad de nutrientes, estimular el crecimiento y las características de floración de plantas de lavanda (Limonium sinuatum, L.).

Memon et al. (2014), investigaron que aplicando $4 \mathrm{~g}$ de ácido húmico + $40 \mathrm{~g}$ de FeSO4 / $2 \mathrm{~m} 2$ de suelo, lograron un nivel óptimo en la producción de flores de zinnia, obteniendo un crecimiento de plantas deseable y altos rendimientos de flores de calidad; en tanto que Parandian y Samavat (2012), probaron que los ácidos húmicos y fúlvicos aumentan significativamente la 


\section{Las sustancias húmicas como sustituto de la materia orgánica en cultivo de rosas (Rosa spp.)}

Vol. 1, núm. 5., (2017)

Víctor Hugo Verdezoto Vargas; Gonzalo Isaías Almagro Mayorga; Iván Edmundo Ramos Mosquera; Ángel Polivio Jines Carrasco; Diego Rivera

cantidad de antocianina en las flores de lilium, presentándose un notable incremento de los azúcares solubles y la enzima $\alpha$-amilasa por un aumento de la cantidad de antocianina en los diferentes tratamientos estudiados.

Elizarrarás et al. (2009), investigaron el establecimiento de una leguminosa forrajera denominada conchita azul (Clitoria ternatea) en un suelo con características homogéneas y predominantemente arcilloso, ésta especie responde bien a la aplicación indistinta de ácidos húmicos (Vermilik y Multiagro) donde estudiaron el comportamiento productivo de C. ternatea para la producción de forraje la cual obtuvo mayor valor con el ácido húmico Vermilik para la variable altura de planta y profundidad de sus raíces.

\section{Conclusión}

Los ácidos húmicos juegan un rol muy importante en el mejoramiento del suelo para que los nutrientes sean mejor aprovechados por las plantas, este efecto se ve irradiado principalmente en cultivos de ciclo corto, por lo que después de realizada esta investigación se llegaron a las siguientes conclusiones:

- Con las aplicaciones de 160 y 200 gramos/m2 de suelo de Humiplex Plus, alcanzaron los valores más altos con 2.25 y 2.03 brotes denominados "basales" respectivamente; los ácidos húmicos estimulan el crecimiento de las raíces y desarrollo de varios órganos de la planta tratada. 


\section{Las sustancias húmicas como sustituto de la materia orgánica en cultivo de \\ rosas (Rosa spp.)}

Vol. 1, núm. 5., (2017)

Víctor Hugo Verdezoto Vargas; Gonzalo Isaías Almagro Mayorga; Iván Edmundo Ramos

Mosquera; Ángel Polivio Jines Carrasco; Diego Rivera

- Usando las mismas dosis anteriores, se alcanzó valores de 1.92 y 1.79 brotes denominados "medias piernas", pues las cifras indican que a medida que se incrementa la dosis de sustancias húmicas (Humiplex Plus) enriquecida con micro elementos (Fe, $\mathrm{Zn}, \mathrm{Mg}, \mathrm{Mn}, \mathrm{Cu}, \mathrm{Bo}$ y S), estimulan el crecimiento y desarrollo de varios órganos de la planta tratada.

- Con los mismos tratamientos anteriores, se alcanzó valores de $36.30 \mathrm{~cm}$ y $35.60 \mathrm{~cm}$ de altura de plantas; estas cifras indican que a medida que se incrementa la dosis de sustancias húmicas/m2 de suelo, también se incrementa la altura de plantas en cultivo de rosas. La aplicación de ácidos húmicos al suelo, mejora el número de botones y la calidad de rosas para el comercio. 


\section{Las sustancias húmicas como sustituto de la materia orgánica en cultivo de rosas (Rosa spp.)}

Vol. 1, núm. 5., (2017)

Víctor Hugo Verdezoto Vargas; Gonzalo Isaías Almagro Mayorga; Iván Edmundo Ramos Mosquera; Ángel Polivio Jines Carrasco; Diego Rivera

\section{Bibliografía}

Ahmad, I., Saquib, R., Qasim, M., Saleem, M., Khan, A. y Yaseen, M. (2013). Humic acid and cultivar effects on growth, yield, vase life, and corm characteristics of gladiolus. Chilean Journal of Agricultural Research, 73(4), 339-344.

Ali, A., Rehman, S., Allah, S, Raza, S. y Allah, S. (2014). Combined effect of Humic Acid and NPK on growth and flower development of Tulipa gesneriana in Faislabad, Pakistan. Journal of Ornamental Plants, 4(4), 39-48.

Álvaro, O. (2008). La materia orgánica (MOS) y su papel en lucha contra la degradación del suelo. XI Congreso Ecuatoriano de la Ciencia del Suelo. Obtenido en http://www.secsuelo.org/wpcontent/uploads/2015/06/9.-Dr.-Alvaro-Garcia.-MO.pdf

Ayón, F., Veliz, D. y Gabriel, J. (2017). El caupí (Vigna unguiculata L. Walp) y su respuesta a la aplicación de ácidos húmicos (AH's) en el Cantón Jipijapa en Ecuador. Journal Of The Selva Andina Biosphere, 5(1), 4-14.

Baldotto, L. y Baldotto, M. (2015). Growth and production of ornamental sunflower grown in the field in response to application of humic acids. Soil Science, 45(5), 1000- 1005.

Bettoni, M., Mógor, Á., Kogerastki, J. y Paulett, V. (2016). Onion (Allium cepa L.) seedling growth using humic substances. Idesia (Arica), 34(2), 57-62.

Canellas, L. y Olivares, F. (2014). Physiological responses to humic substances as plant growth promoter. Chemical and Biological Technologies in Agriculture, 1(3), 1-11.

Chang, L., Wu, Y., Xu, W., Nikbakht, A. y Xia, Y. (2012). Effects of calcium and humic acid treatment on the growth and nutrient uptake of Oriental Lily. African Journal of Biotechnology, 11(9), 2218-2222.

Elizarrarás, S., Serrato, J. y López, E., (2009). La aplicación de ácidos húmicos en las características productivas de Clitoria ternatea L. en la región Centro Occidente de México. Revista avances en investigación agropecuaria, 13(3) ,11-15.

FAO. (2013). El manejo del suelo en la producción de hortalizas con buenas prácticas agrícolas. Obtenido en http://www.fao.org/3/a-i3361s.pdf

Gobierno autónomo descentralizado de la parroquia de Otón. (2014). Actualización del plan de desarrollo y ordenamiento territorial (PDOT). Obtenido en http://app.sni.gob.ec/sni- 


\section{Las sustancias húmicas como sustituto de la materia orgánica en cultivo de rosas (Rosa spp.)}

Vol. 1, núm. 5., (2017)

Víctor Hugo Verdezoto Vargas; Gonzalo Isaías Almagro Mayorga; Iván Edmundo Ramos Mosquera; Ángel Polivio Jines Carrasco; Diego Rivera

link/sni/PORTAL_SNI/data_sigad_plus/sigadplusdiagnostico/1768107850001_PDYOT\%20 OTON_30-10-2015_21-56-30.pdf

González, M., Jiménez, L., Yánez, W. y Parducci, P. (2018). Potencial uso de la leonardita para el cultivo de rosa en condiciones de invernadero. Agronomía Costarricense, 42(1), 155-162.

Gutiérrez, J., González, G., Segura, M., Sánchez, I., Orozco, J. y Fortis, M. (2015). Efecto de ácidos húmicos de Leonardita en la estabilidad de agregados del suelo y raíces de melón en condiciones de invernadero. Revista Internacional de Botánica Experimental, 84(2), 298-305.

Hernández, R., García, A., Portuondo, L., Muñiz, S., Berbara, R. he Izquierdo, F. (2012). Protección antioxidativa de los ácidos húmicos extraídos de vermicompost en arroz (Oryza sativa L.) var. IACuba30. Revista de Protección Vegetal, 27(2), 102-110.

Huelva, R., Martínez, D., Calderín, A., Hernández, O. y Guridi, F. (2013). Propiedades químicas y química-físicas de derivados estructurales de ácidos húmicos obtenidos de vermicompost. Actividad biológica. Revista Ciencias Técnicas Agropecuarias, 22(2), 56-60.

Ibrahim, H., Fadaly, H. y Naggar, A. (2016). Study on the Response of Statice Plants (Limonium sinuatum, L.) to Humic Acid Application. Alexandria Science Exchange Journal, 37(3), 515528.

Janampa, N., Quiñones, A., Suárez, L. y Chalco, Y. (2014). Variación de sustancias húmicas de abonos orgánicos en cultivos de papa y maíz. Ciencias del suelo, 32(1), 139-147.

Kulikova, N., Abroskin, D., Badun, G., Chernysheva, M., Korobkov, V., Beer, A., Tsvetkova, E., Senik, S., Klein, O. y Perminova, I. (2016). Label Distribution in Tissues of Wheat Seedlings Cultivated with Tritium-Labeled Leonardite Humic Acid. Scientific Reports, 6, 28869.

Manda, M., Dumitru, M. y Nicu, C. (2014). Effects of humic acid and grape seed extract on growth and development of Spathiphyllum wallisii Regel. South Western Journal of Horticulture, Biology and Environment, 5 (2), 125-136.

Memon, S., Bangulzai, F., Keerio, M., Baloch, M. y Buriro, M. (2014). Effect of humic acid and iron sulphate on growth and yield of zinnia (Zinnia elegans). Journal of Agricultural Technology, 10(6), 1517-1529.

Mindari, W., Aini, N., Kusuma, Z. y Syekhfani. (2014). Effects of humic acid-based buffer + cation on chemical characteristics of saline soils and maize growth. Journal of degraded and mining lands management, 2, 259-268. 


\section{Las sustancias húmicas como sustituto de la materia orgánica en cultivo de rosas (Rosa spp.)}

Vol. 1, núm. 5., (2017)

Víctor Hugo Verdezoto Vargas; Gonzalo Isaías Almagro Mayorga; Iván Edmundo Ramos Mosquera; Ángel Polivio Jines Carrasco; Diego Rivera

Noriega, B., Cortes, E., Gómez, M., Ramírez, D. y Echeverría, C. (2015). Evaluación de ácidos húmicos en el cultivo de la rosa (Rosa sp.) en Temixco, Morelos: $11^{\circ}$ Foro Estatal y $3^{\circ}$ Foro Regional de Investigación y experiencias educativas y productivas (en línea). Disponible en http://www.cbta39.edu.mx/sites/default/files/foro\%202015_EJEMPLO\%201\%20RESUM EN.pdf

Pablo, C., Martínez, E., Rosas, I., Hernández, M. y Ponce, C. (2015). Caracterización de la materia orgánica en sedimentos de los canales de Xochimilco. 1er Congreso Iberoamericano sobre sedimentos y ecología.

Parandian, F. y Samavat, S. (2012). Effects of Fulvic and Humic acid on Anthocyanin, soluble Sugar, Amylase Enzyme and some micronutrient elements in Lilium. Intl. International Research Journal of Applied and Basic Sciences, 3(5), 924-929.

Potisek, M., González, G., Velásquez, M. y Macías, H. (2013). Efecto del ácido fúlvico en la extracción nutrimental y rendimiento del cultivo de melón en bioespacio. AGROFAZ, 13(2), 23-29.

Reyes, J., Abasolo, F., Yépez., Á., Luna, R., Zambrano D., Vázquez, V., Cabrera, D., Guzmán, J., Torres, J. y Rodríguez, W. (2017). Ácidos húmicos y su efecto sobre variables morfométricas en plantas de zanahoria (Daucus carota L). Biotecnia, 19(2), 25-29.

Ramírez, F., Gómez, J. y Flórez, V. (2011). Evaluación del Fertilizante Orgánico Líquido de Lombriz San Rafael en el Cultivo de Rosa cv. Classy. Revista Facultad Nacional de Agronomía Medellín, 64(2), 6147-6157.

Túqueres, M. (2013). Respuesta del cultivo de alstroemerias (Alstroemeria aurantiaca) a la aplicación de tres dosis de ácidos húmicos y ácidos fúlvicos, en el mejoramiento de la producción, en la zona de Cayambe, provincia de Pichincha

Velasco, J. Aguirre, G y Ortuño, N. (2016). Humus líquido y microorganismos para favorecer la producción de lechuga (Lactuca sativa var. Crespa) en cultivo de hidroponía. Journal Of The Selva Andina Biosphere, 4(2), 71-83. 